\title{
Plume Grain Sampling at Hypervelocity: Implications for Astrobiology Investigations
}

\author{
A Science White Paper for the Decadal Survey
}

\section{Authors and Affiliations:}

Morgan L. Cable, NASA Jet Propulsion Laboratory, California Institute of Technology Sarah E. Waller, NASA Jet Propulsion Laboratory, California Institute of Technology Rob Hodyss, NASA Jet Propulsion Laboratory, California Institute of Technology Amy E. Hofmann, NASA Jet Propulsion Laboratory, California Institute of Technology Michael J. Malaska, NASA Jet Propulsion Laboratory, California Institute of Technology Robert E. Continetti, University of California, San Diego Andres Jaramillo-Botero, California Institute of Technology Bernd Abel, University of Leipzig Frank Postberg, Freie Universität Berlin Morgan E. C. Miller, University of California, San Diego Sally Burke, University of California, San Diego Anton Belousov, NASA Jet Propulsion Laboratory, California Institute of Technology Fabian Klenner, Freie Universität Berlin Nick Tallarida, NASA Jet Propulsion Laboratory, California Institute of Technology James Lambert, NASA Jet Propulsion Laboratory, California Institute of Technology Steve Fuerstenau, Radmet Inc.

Zach Ulibarri, Center for Integrated Plasma Studies, University of Colorado

Primary Author Contact: Morgan.L.Cable@jpl.nasa.gov

\section{Endorsers:}

Sean Hsu, Laboratory for Atmospheric and Space Physics, University of Colorado, Boulder Susan Lang, University of South Carolina Ralf Srama, University of Stuttgart, Stuttgart, Germany

Sascha Kempf, Laboratory for Atmospheric and Space Physics, University of Colorado, Boulder Everett L. Shock, Arizona State University Linda J. Spilker, NASA Jet Propulsion Laboratory, California Institute of Technology John Robert Brucato, INAF-Astrophysical Observatory of Arcetri, Firenze Italy

A portion of this research was carried out at the Jet Propulsion Laboratory, California Institute of Technology, under a contract with the National Aeronautics and Space Administration (80NM0018D0004). (C) 2020. All rights reserved. 


\section{Summary}

Mass spectrometry (MS) using impact-induced ionization is an effective means of detecting organic molecules entrained within ice grains. Recent work has demonstrated that the optimal speed for volatilizing and ionizing biomolecules of interest in ice grains is $4-6 \mathrm{~km} / \mathrm{s}$. Above this speed range, the biomolecules start to fragment; below this threshold, the energy is not sufficient to promote ionization. A spacecraft with an impact plate and mass spectrometer could therefore be used to perform astrobiology investigations at Enceladus or possibly Europa, without the cost of resources to slow down and/or land. We recommend support of further experimental and theoretical work, in particular a focus on impacts of neutral ice grains, in order to gain a deeper understanding of the physical and chemical mechanisms that occur during hypervelocity impact. We also recommend investment in developing the next generation of MS techniques for hypervelocity sampling, as well as producing/generating mass spectral libraries for future missions to use in the identification of organics and biosignatures.

\section{Introduction}

The 2019 Astrobiology Strategy of the National Academies describes a chemical biosignature as "the presence of specific patterns in the abundances of selected compounds that do not typically occur via chance in reactions driven by thermodynamics alone" (National Academies, 2019). Detection of a chemical biosignature therefore requires unambiguous identification and quantitation of these compounds. Detection of agnostic biosignatures (i.e., those that do not presuppose any particular molecular framework, such as the phosphate backbone in DNA) also requires specific molecular identification in order to determine the number of pathways by which that molecule could be assembled (Cronin and Walker, 2016). The goal of this white paper is to provide an update on the latest experimental and theoretical work regarding hypervelocity sampling for astrobiology investigations, and to identify areas in need of further investment.

In this white paper, we address the following question: can astrobiology investigations be made at hypervelocity, i.e., does biosignature detection have a speed limit?

Hypervelocity typically refers to speeds in excess of $3 \mathrm{~km} / \mathrm{s}$ (Torvik, 1991). During impacts at these velocities, virial atomic stresses are high enough to dissociate the chemical bonds that hold the material together. Hypervelocity impacts occur regularly in the space environment, and can lead to damage of spacecraft surfaces and weathering of airless bodies (moons, comets, etc.). However, hypervelocity impacts can also be utilized to our advantage: the NASA Discovery mission Deep Impact collided with comet Tempel 1 at $10 \mathrm{~km} / \mathrm{s}$ and was the first mission to probe the interior of a comet, with discoveries that included the detection of polycyclic aromatic hydrocarbons (Henderson and Blume, 2015; Lisse et al., 2006).

Many missions involve flyby or orbital encounters with planetary bodies at hypervelocity. In situ measurements are not precluded at these speeds-both the Galileo and Cassini spacecraft had dust detectors capable of measuring small particles traveling at velocities of 1-70 km/s and 2-40 km/s, respectively (Grün et al., 1992; Srama et al., 2004). Both missions also had neutral mass spectrometers capable of measuring gases in the atmospheres of Jupiter (via the entry probe) and Titan (during a flyby) at similar velocities, as the ram pressure of inflowing gas enhances the density of constituents in a known way that can be effectively modeled (Magee et al. 2009). 
Cassini had the unique opportunity to sample the plume grains of Enceladus at hypervelocity (7$17 \mathrm{~km} / \mathrm{s}$ ) during multiple flyby encounters. The Cosmic Dust Analyzer (CDA) aboard Cassini was able to detect large (>200 u) organic molecules at encounter speeds in excess of $7 \mathrm{~km} / \mathrm{s}$ (Postberg et al., 2018). These chemically diverse macromolecules were sourced from organic-rich (>1 wt\%) ice grains that volatilized and generated ions upon impact with the rhodium plate of the instrument. The mass spectral peaks of these organic-rich ice grains showed a pattern (a monotonic decrease without major intensity variations) that indicates the organics may be fragments of even larger molecules, as opposed to a conglomerate of several molecules with masses below $200 \mathrm{u}$. This suggests that impact speeds $<7 \mathrm{~km} / \mathrm{s}$ might be needed to sample most of the parent molecules. If that is the case, what is the proper speed at which to perform astrobiology investigations - or other analyses of large, complex organic molecules - from orbital or flyby missions?

The answer depends on what one wishes to do with the collected sample. Various capture materials exist for collecting grains at hypervelocity. Aerogel is the most common; it is comprised of a low density matrix that slows the particles down gradually, preserving their composition (Burchell et al., 2010). However, extracting the particles later can be difficult (this typically requires manual removal) and aerogel is challenging to manufacture and maintain free of organic contamination (Sandford et al. 2010) which could make interpretation of an astrobiology measurement difficult (Elsila et al. 2008). Other technologies for collecting hypervelocity samples include accommodation chambers such as the closed source of the Cassini lon and Neutral Mass Spectrometer (INMS), and funnels comprised of highly ordered surface materials (Murray et al., 2017). However, most of these are designed for sampling atmospheres as opposed to ice grains in a plume. In this white paper we focus on a simpler solution that minimizes contamination while enabling in situ analysis of ice grains.

\section{Impact-Induced Ionization: A Simpler Solution}

To avoid potential issues with sample extraction/handling and contamination, many instruments aboard past and planned missions (including but not limited to Galileo, Cassini, and Europa Clipper) were designed to interrogate ice grains instantaneously by using the hypervelocity impact itself to induce ionization. This process, called impact-induced ionization, utilizes the kinetic energy of a particle's impact to volatilize and ionize species within the particle. When an ice grain of sufficient speed hits a hard surface (e.g., $\mathrm{TiO}_{2}$ ), it is vaporized. (In extreme conditions, a small percentage of the surface can also be affected (Jaramillo-Botero et al., 2012).) Some of the initial kinetic energy also ionizes the vaporized material, thereby generating a plasma. The resulting ions can then be directly analyzed with a mass spectrometer.

The impact velocity must be tuned to optimize the impact-induced ionization efficiency. If the velocity is too fast, severe fragmentation of molecules will occur, which can confound identification and quantification of the original molecules present. If the impact velocity is carefully chosen, the chemical constituents of a grain can be volatilized, and their valence electrons ionized, without inducing significant fragmentation. Impact-induced ionization in the appropriate velocity regime is the simplest way to ionize ice grain constituents for subsequent MS analysis, while preserving the identity of the parent species.

Impact-induced ionization is a simple, effective, and commonly used technique to ionize molecules for analysis with mass spectrometry while minimizing contamination. 
Cassinis CDA utilized impact-induced ionization in conjunction with a time of flight (TOF) mass spectrometer, as will the SUrface Dust Analyzer (SUDA) aboard Europa Clipper. Mass spectrometers are excellent tools for compositional measurements, as they respond to nearly all types of molecules in the sample. Cassinis CDA identified water-ice, salts and a plethora of organic molecules in the ice grains emanating from Enceladus. Future missions to Enceladus have been proposed that would implement a similar approach to search for evidence of biosignatures in the plume (Reh et al. 2016). However, for astrobiology investigations, preserving the structure of organic molecules is critical, and in the past it was unclear if impact-induced ionization would be 'soft' enough to keep large organic molecules intact. In the sections that follow, we provide an update on the latest experimental and theoretical work regarding hypervelocity sampling for astrobiology investigations, and identify areas in need of further investment.

\section{Finding the Speed Limit of Biomolecules: Experimental and Theoretical Work}

Since the arrival of Cassini in the Saturn system in 2004, scientists have worked in the laboratory to replicate data produced by this spacecraft's instruments to improve interpretation of results. For the CDA instrument, the method that most accurately reproduces ice grain impact mass spectra is a laser-based analogue experiment. A micron-diameter water beam is intersected with a pulsed IR laser in a vacuum, which generates an explosive dispersion that produces atomic, molecular and macroscopic fragments (Charvat et al. 2007). Charged species are analyzed using a reflectron time-of-flight (TOF) mass spectrometer. Using this technique, the CDA Science Team was able to identify and quantify salts and organic molecules-including high-mass organic molecules with heteroatoms-in the Enceladus plume grains (Postberg et al. 2009, 2011, 2018; Khawaja et al. 2019). They have also recently been able to predict optimal encounter velocities for biomolecules of interest. By varying the laser intensity and delay time, different ice grain impact velocities can be simulated in the laser desorption process. For amino acids, the sensitivity of a MS instrument using impact-induced ionization is maximized at speeds of $4-10 \mathrm{~km} / \mathrm{s}$; for fatty acids, the optimal speed range is 3-6 km/s (Klenner et al., 2020).

Theoretical modeling work also supports these velocities. High-fidelity simulations of the fragmentation of amino and fatty acids were performed using a first-principles based approach, and found that bare molecules (e.g., amino acids such as arginine and fatty acids such as palmitic acid) typically begin to fragment at $3-5 \mathrm{~km} / \mathrm{s}$, whereas those same molecules entrained within ice grains fragment at higher velocities $(4-6 \mathrm{~km} / \mathrm{s})$ due to dissipation of energy within the ice shell (Jaramillo-Botero et al., 2020). The relatively narrow impact velocity threshold window in which amino and fatty acids start to fragment suggests that sampling at the single upper limit velocity for this group of biosignature molecules $(6 \mathrm{~km} / \mathrm{s})$ would enable deconvolution of the mass spectra, without compromising the signatures of the parent molecules.

Experiments and theoretical models made to reproduce Cassini data suggest that the optimal speed to sample amino acids and fatty acids in ice grains is $4-6 \mathrm{~km} / \mathrm{s}$.

\section{Ongoing and Future Work to Validate Astrobiology at Hypervelocity}

The recommended hypervelocity sampling speed limit for biomolecules has been set by experiments that reproduce Cassini CDA data, and this is supported by theoretical work. However, it has not yet been validated by impacting an ice grain of Enceladus-appropriate size (1.2 $\mu \mathrm{m}$ avg diameter) and composition (1 wt\% organics) in a laboratory setting at $4-6 \mathrm{~km} / \mathrm{s}$. 
The light gas gun at the University of Kent is capable of firing a hollowed-out sabot $(4.5 \mathrm{~mm}$ diameter, $6.0 \mathrm{~mm}$ length) containing water-ice doped with organics or cells at velocities up to 7.4 $\mathrm{km} / \mathrm{s}$ (Price et al. 2013). Organic ice-particle simulants (polymethylmethacrylate) 4-10 $\mu \mathrm{m}$ in diameter were also fired up to $3.0 \mathrm{~km} / \mathrm{s}$ using a sabot discarded in flight in this gas gun (New et al. 2020). However, these experiments are not performed in a vacuum and the impacted grains cannot be studied in situ, as they must be extracted from the chamber before analysis.

The dust accelerator facility at the Institute for Modeling Plasma, Atmospheres, and Cosmic dusT (IMPACT) at the University of Colorado at Boulder has developed a 'reciprocal experiment', in which micron-sized Fe or Al particles are impacted into a cryocooled water-ice surface at 3-50 $\mathrm{km} / \mathrm{s}$ and the resulting impact-induced ions are analyzed using a TOF mass spectrometer. Recent data indicate that amino acids and polypeptides remain intact, and-even after fragmentation of these biomolecules at higher velocities-the products can be used to identify the original constituents in certain cases (Ulibarri et al., 2019). However, the absorption of energy across a flat ice surface may not be representative of energy absorption by a micron-diameter ice grain.

Current work is underway to accelerate ice grains of Enceladus-relevant size (microns) and composition (water-ice with salts and/or organics) in a vacuum up to hypervelocity. The Aerosol Impact Spectrometer (AIS) at the University of California, San Diego is capable of trapping a single ice grain $(0.1-2 \mu \mathrm{m})$, characterizing its size and charge, accelerating it to speeds $>2 \mathrm{~km} / \mathrm{s}$ using a series of linear acceleration stages, and characterizing post-impact ions using a TOF mass spectrometer (Miller et al. 2019). Modifications are being made to increase the capability of this system to $5 \mathrm{~km} / \mathrm{s}$. A separate system is also under development at the Jet Propulsion Laboratory (JPL) to impact a distribution of ice grains onto a metal plate at $3-5 \mathrm{~km} / \mathrm{s}$ and analyze the ions produced with an orthogonal TOF mass spectrometer. Both systems should be able to experimentally reproduce the impact of an ice grain during sampling by a spacecraft in a plume flythrough encounter, and will hopefully confirm the 4-6 km/s speed limit established by theory and CDA analogue experiments.

Work is underway to more accurately reproduce the impact of an ice grain laden with biomolecules in the laboratory. These systems will represent the new state-of-the-art for astrobiology investigations performed at hypervelocity for Enceladus and possibly Europa.

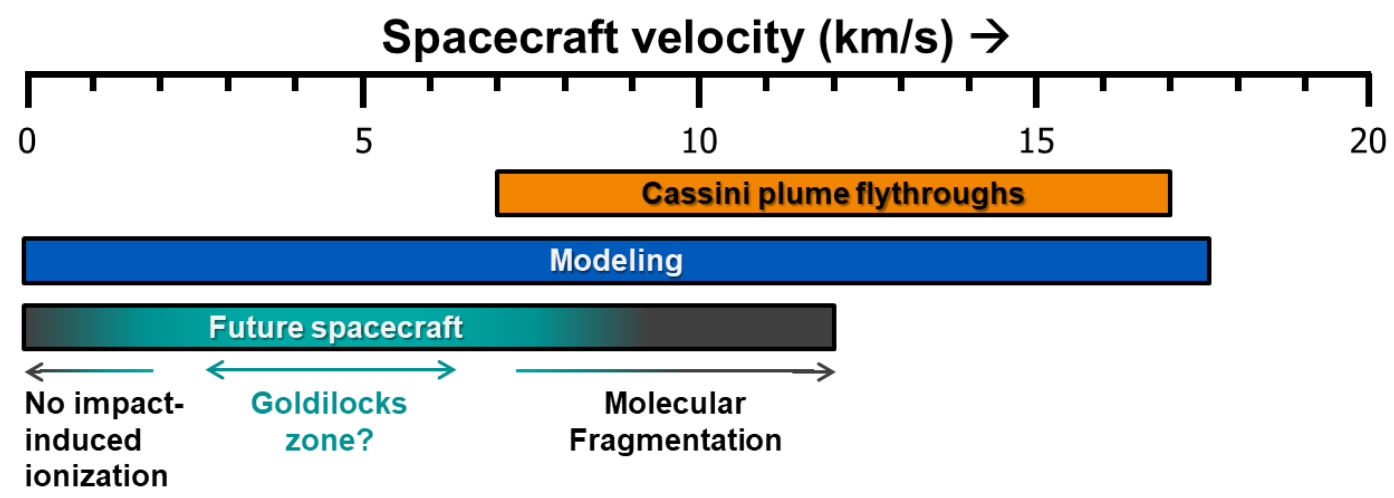

Figure 1. A future astrobiology investigation targeting ice grains of Enceladus or Europa at hypervelocity would do so at a speed fast enough to ionize the biomolecules inside the grains, but slow enough to avoid severe fragmentation of these molecules. Figure by $\mathrm{S}$. Waller. 


\section{Recommendations}

While significant work has been done or is in development to understand the physics and chemistry behind hypervelocity impacts of molecules and organic-laden ice grains, limitations still exist. Most experimental systems that reach the appropriate size range of ice grains (microns) can only accelerate grains that carry a charge, or a mixture of charged and neutral species. We recommend that NASA invest in:

- Experimental facilities that can simulate hypervelocity impacts of only neutral molecules/grains. These facilities could enable evaluation of the differences between the charged versus neutral impact processes, as well as permit testing and calibration of flight instruments and development of new strategies for mitigation of hypervelocity effects. Such work would enable more capable science investigations-in particular those focused on identification and quantification of the organic inventory of a plume or atmosphere - to be included on flyby and orbital missions at lower cost and complexity compared to landed architectures.

- Theoretical/computational methods for elucidating the mechanisms of impact-induced ionizing fragmentation of molecules, and for simulating the long-term (i.e., over the full timescale of post-impact chemistry) dynamics of hypervelocity impacts of molecules and particles. These methods, including non-adiabatic capabilities, would provide theoretical grounding for the observed phenomena.

- The next generation of mass spectral techniques for hypervelocity sampling, including pursuing capture technologies (e.g. funnels) that could expand the range of velocities available for astrobiology investigations.

- Benchmarking simulations with experimental data to allow extrapolation to conditions, molecules, and reactive intermediates that are difficult or in some cases impossible to obtain in a laboratory setting.

- Generating a library of fragmentation pathways for possible biosignature molecules that would facilitate astrobiology-focused mission interpretation.

These investments would improve the ability to interpret measurements made at hypervelocity, both by past and future missions, and lead to a better understanding of the mechanisms of impact fragmentation for the specific conditions a particular spacecraft is likely to encounter.

NASA should continue to invest in facilities capable of simulating ice grain impacts at hypervelocity, including neutral grains and theoretical models. NASA should also invest in the development of mass spectral libraries to better interpret data from MS instruments.

\section{Conclusion}

Many important science questions, including those pertaining to astrobiology and the organic chemistry of the subsurface ocean of Enceladus, can be effectively addressed with mission concepts that sample materials at hypervelocity. While the optimal speed for analyzing biomolecules entrained within ice grains is still under investigation, experimental and theoretical work indicate $3-5 \mathrm{~km} / \mathrm{s}$ as the right speed range to ionize complex bare organic molecules, and 4-6 km/s if they are entrained in ice clusters/grains, upon impact without fragmentation of the parent species. Further experimental and theoretical work, in particular concerning neutral grain impacts, theoretical and computational methods to understand impact dynamics and 
fragmentation pathways, as well as exploring the next generation of MS techniques, would help us better understand the mechanisms behind ionization, fragmentation, and chemistry at hypervelocity.

Astrobiology investigations do have a speed limit: $3-5 \mathrm{~km} / \mathrm{s}$ for bare molecules, $4-6 \mathrm{~km} / \mathrm{s}$ for biomolecules in ice grains. Spacecraft with MS instruments at these speeds could address questions of life detection at Enceladus and possibly Europa. NASA should invest in further laboratory and theoretical studies to increase the scientific return of upcoming and proposed missions equipped with these instruments.

\section{Abbreviations}

AIS, Aerosol Impact Spectrometer; CDA, Cosmic Dust Analyzer; IMPACT, the Institute for Modeling Plasma, Atmospheres, and Cosmic dusT; INMS, Ion and Neutral Mass Spectrometer; LASP, Laboratory for Atmospheric and Space Physics; MS, Mass Spectrometry; SUDA, SUrface Dust Analyzer; TOF, Time-Of-Flight.

\section{References}

Burchell, M. J. et al. (2010) Capture of particles in hypervelocity impacts in aerogel. Meteor. Planet. Sci, 36 (2), 209-221. https://doi.org/10.1111/j.1945-5100.2001.tb01865.x.

Cable, M. L., M. Neveu, H.-S. Hsu and T. Hoehler (2019) "Enceladus" in Planetary Astrobiology, Eds. V. Meadows et al. Space Science Series. Univ. of Arizona Press and LPI. In press.

Charvat, A. and B. Abel (2007) How to make big molecules fly out of liquid water: applications, features and physics of laser assisted liquid phase dispersion mass spectrometry. Phys. Chem. Chem. Phys., 9 (26), 3335-3360. https://doi.org/10.1039/B615114K

Cronin, L. and S. I. Walker (2016) Beyond prebiotic chemistry. Science, 352 (6290), 1174-1175.

Elsila, J. E., D. P. Glavin, and J. P. Dworkin (2008) Cometary glycine detected in samples returned by Stardust. Meteoritics and Planetary Science, 44 (9), 1323-1330.

Grün, E. et al. (1992) The Galileo Dust Detector. Space Sci. Rev., 60, 317-340.

Henderson, M. et al. (2015) Deep Impact - A Review of the World's Pioneering Hypervelocity Impact Mission. Proc. Eng., 103, 165-172. https://doi.org/10.1016/i.proeng.2015.04.023

Jaramillo-Botero, A., M. L. Cable, A. E. Hofmann, et al. (2020) Understanding hypervelocity sampling of biosignatures in space missions. Astrobiology, submitted.

Jaramillo-Botero, A., et al. (2012) Hypervelocity impact effect of molecules from Enceladus' Plume and Titan's upper atmosphere on NASA's Cassini Spectrometer from Reactive Dynamics Simulations. PRL, 109, 213201. https://doi.org/10.1103/PhysRevLett.109.213201

Khawaja, N., F. Postberg, J. Hillier, et al. (2019) Low-mass nitrogen-, oxygen-bearing, and aromatic compounds in Enceladean ice grains. Mon. Not. R. Astron. Soc., 489 (4), 52315243. https://doi.org/10.1093/mnras/stz2280

Klenner, F., F. Postberg, J. Hillier, et al. (2020) Discriminating Abiotic and Biotic Fingerprints of Amino Acids and Fatty Acids in Ice Grains Relevant to Ocean Worlds. Astrobiology, ahead of print. http://doi.org/10.1089/ast.2019.2188 
Lisse, C. M., J. VanCleve, A. C. Adams et al. (2006) Spitzer spectral observations of the Deep Impact ejecta. Science, 313 (5787), 635-640. https://doi.org/10.1126/science.1124694.

Magee, B. A., J. H. Waite, K. E. Mandt et al. (2009) INMS-derived composition of Titan's upper atmosphere: Analysis methods and model comparison. Planet. Space Sci., 57, 14-15, 18951916. https://doi.org/10.1016/i.pss.2009.06.016.

Miller, M. E. C., et al. (2019) Hypervelocity Enceladus ice grain analogue production with the Aerosol Impact Spectrometer. EPSC-DPS Joint Meeting, 15-20 Sept 2019, Vol. 13. https://meetingorganizer.copernicus.org/EPSC-DPS2019/EPSC-DPS2019-353-1.pdf

Murray, V. J. et al. (2017) Gas-surface scattering dynamics applied to concentration of gases for mass spectrometry in tenuous atmospheres. J. Phys. Chem. C, 121 (14), 7903-7922. https://doi.org/10.1021/acs.jpcc.7b00456.

National Academies of Sciences, Engineering, and Medicine (2019) An Astrobiology Strategy for the Search for Life in the Universe. Washington, D.C.: The National Academies Press. https://doi.org/10.17226/25252

New, J. S., et al. (2020) Characterizing organic particle impacts on inert metal surfaces: Foundations for capturing organic molecules during hypervelocity transits of Enceladus plumes. Meteorit. Planet. Sci., 55 (3), 465-479. https://doi.org/10.1111/maps.13448

Postberg F., et al. (2009) Sodium salts in E-ring ice grains from an ocean below the surface of Enceladus. Nature, 459, 1098-1101. https://doi.org/10.1038/nature08046

Postberg F., et al. (2011) A salt-water reservoir as the source of a compositionally stratified plume on Enceladus. Nature, 474, 620-622. https://doi.org/10.1038/nature10175

Postberg, F., N. Khawaja, B. Abel, et al. (2018) Macromolecular organic compounds from the depths of Enceladus. Nature, 558, 564-568. https://doi.org/10.1038/s41586-018-0246-4.

Price, M. C., et al. (2013) Survival of yeast spores in hypervelocity impact events up to velocities of $7.4 \mathrm{~km} \mathrm{~s}^{-1}$. Icarus, 222 (1), 263-272. https://doi.org/10.1016/.icarus.2012.10.035.

Reh, K., et al. (2016) Enceladus Life Finder: The search for life in a habitable moon. IEEE Aerospace Conference, 5-12 Mar 2016, DOI: 10.1109/AERO.2016.7500813.

Sandford, S. A., et al. (2010) Assessment and control of organic and other contaminants associated with the Stardust sample return from comet 81P/Wild 2. Meteoritics and Planetary Science, 45 (3), 406-433. https://doi.org/10.1111/j.1945-5100.2010.01031.x.

Srama, R., (2004) "The Cassini Cosmic Dust Analyzer." In: Russell C. T. (eds) The CassiniHuygens Mission. Springer, Dordrecht. https://doi.org/10.1007/978-1-4020-2774-1 7.

Torvik, P. J. (1991) Hypervelocity Projectiles. In Critical Technologies for National Defense, Przemieniecki, J. S., Ed. American Institute of Aeronautics and Astronautics: Washington, D.C., 287-306.

Ulibarri, Z., T. L. Munsat, B. Abelet al. (2019) On the Genesis and Detectability of Organic Chemistry in Hypervelocity Impact Ice Spectra. AGU Fall Meeting, abstract \#P43C-3485. https://ui.adsabs.harvard.edu/\#abs/2019AGUFM.P43C3485U/abstract 